\title{
A preliminary taxonomy of medical errors in family practice
}

\author{
S M Dovey, D S Meyers, R L Phillips Jr, L A Green, G E Fryer, J M Galliher, J Kappus, \\ P Grob
}

See end of article for authors' affiliations

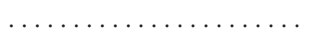

Correspondence to: Dr S Dovey, The Robert Graham Center: Policy Studies in Family Practice and Primary Care, 2023 Massachusetts Ave NW, Washington, DC 20036 USA; sdovey@aafp.org

Accepted for publication 22 May 2002

\begin{abstract}
Objective: To develop a preliminary taxonomy of primary care medical errors.
Design: Qualitative analysis to identify categories of error reported during a randomized controlled trial of computer and paper reporting methods.

Setting: The National Network for Family Practice and Primary Care Research.

Participants: Family physicians.

Main outcome measures: Medical error category, context, and consequence.

Results: Forty two physicians made 344 reports: 284 (82.6\%) arose from healthcare systems dysfunction; $46(13.4 \%)$ were errors due to gaps in knowledge or skills; and $14(4.1 \%)$ were reports of adverse events, not errors. The main subcategories were: administrative failures $1102 ; 30.9 \%$ of errors), investigation failures $(82 ; 24.8 \%)$, treatment delivery lapses $(76 ; 23.0 \%)$, miscommunication $(19 ; 5.8 \%)$, payment systems problems $(4 ; 1.2 \%)$, error in the execution of a clinical task $(19 ; 5.8 \%)$, wrong treatment decision $(14 ; 4.2 \%)$, and wrong diagnosis $(13 ; 3.9 \%)$. Most reports were of errors that were recognized and occurred in reporters' practices. Affected patients ranged in age from 8 months to 100 years, were of both sexes, and represented all major US ethnic groups. Almost half the reports were of events which had adverse consequences. Ten errors resulted in patients being admitted to hospital and one patient died.

Conclusions: This medical error taxonomy, developed from self-reports of errors observed by family physicians during their routine clinical practice, emphasizes problems in healthcare processes and acknowledges medical errors arising from shortfalls in clinical knowledge and skills. Patient safety strategies with most effect in primary care settings need to be broader than the current focus on medication errors.
\end{abstract}

$\mathrm{P}$ rimary care is characterized by customized care that responds to individual patients' needs, values, and preferences across a broad spectrum of health care..$^{1-3}$ Its diversity, scope, and variation in structure and infrastructure may offer more opportunity for error than more highly regulated and procedure oriented hospital based care.

Research into medical errors in hospitals $s^{4-14}$ has identified and proposed remedies to significant threats to patient safety in anaesthetics, ${ }^{12}$ medication use, ${ }^{511}{ }^{13}$ and perioperative and intraoperative care, ${ }^{14}$ but many of these lessons cannot readily be translated into primary care. While hospital care clearly exposes patients to certain types of errors that could cause substantial harm (such as wrong side surgery ${ }^{4}$ ), hospitals are also the least frequently used component of formal health systems for most people. ${ }^{15}{ }^{16}$ Important opportunities for protecting patients from harm may exist in other settings.

Making primary care safer for patients is complicated by a lack of understanding of the nature and distribution of errors that occur in this setting. Only two studies have explored the epidemiology of threats to patient safety in primary care. ${ }^{17-19}$ One described "critical incidents" that were not necessarily errors, ${ }^{17}{ }^{18}$ while the other examined the records of a risk management database ${ }^{19}$ using a taxonomy developed from a review of hospital records. ${ }^{4}$

In 2000 we conducted a trial of computer and paper error reporting systems. Data collected for this study included detailed reports of medical errors noticed by family physicians in clinical practice. The purpose of the current investigation was to use these data to develop a preliminary taxonomy of medical errors observed by family physicians in their daily practice.

\section{METHODS}

\section{Study participants}

Forty two family physician members of the American Academy of Family Physicians' (AAFP) National Network for Family Practice and Primary Care Research (the National Network) volunteered to participate in the study. They were evenly distributed throughout the US ( 10 in the north east, 12 in the south, nine in the mid-west, and 11 in the west) and were similar to all 64714 non-federal US family physicians in mean age (study physicians 46 years, all family physicians 48 years), sex (75.7\% $v 75.9 \%$ male), and mean years in medical practice ( 16 years $v 17$ years). All participants were board certified in family practice compared with $86.3 \%$ of active AAFP family physician members. Most $(73.0 \%)$ did not use electronic medical records in their practice and most $(83.8 \%)$ did not have access to the Internet.

\section{Study design}

Data were collected as part of a crossover randomized controlled trial of paper and computer reporting systems in which physicians made anonymous reports by both methods. Paper reports were made using a traditional card based data collection system ${ }^{1620}$ and computer reports were made using an error reporting tool designed for the study by the World Health Network (http://www.healix.com). Paper reports were sent to the National Network office in Kansas where they were stripped of identifiers and assigned a numeric code to signify the physician making the report. Electronic data were sent encrypted from physicians' practices in the US through the secure World Health Network server in London before also being sent to the Kansas office (see box 1 for further technical 
Box 1 Data transmission processes

Electronic data were gathered by a Healix SmartForm solution provided by World Health Network. Data were collected on doctors' desktop computers and required an Internet connection only when a batch of reports was ready for sending. Reports were collected and collated by secure servers in the UK, automatically decrypted, renumbered, and re-encrypted with unique keys for onward transmission to the American Academy of Family Physicians headquarters in Kansas and from there to the Robert Graham Center in Washington DC. Recent software developments provide research data recipients with the choice of receiving data via email or special robots that periodically dial up and retrieve the latest data and populate a database. When this study was conducted, email transfer was the only option. The Healix system was fully automated and required no technical knowledge to implement. Data were fully encrypted during transmission and decoded only after receipt by the American Academy of Family Physicians. The data were anonymous apart from the identity numbers issued by the National Network office.

details). Reports were then sent to the Robert Graham Center: Policy Studies in Family Practice and Primary Care (the Robert Graham Center) in Washington, DC. The process resulted in a set of anonymous reports that could be associated with a unique participating physician.

The Georgetown University Institutional Review Board approved the study. During data collection, all error reports were reviewed in the Robert Graham Center by a family physician (LG) who was not involved in data analysis, and a process was created to contact participating physicians to resolve situations where patients potentially remained at risk of harm. No such situation arose.

\section{Study data}

The study was planned to produce sufficient qualitative data to give depth and credibility to the characterization of errors in a preliminary taxonomy. After discussion with National Network physicians, we concluded: (1) that 500 error reports would provide sufficient data to derive a valid characterization of the errors recognized and reported in family practice; (2) that recruitment of approximately 50 physicians would give sufficient diversity of practice locations and settings to ensure that the study data were not unduly influenced by a few prolific reporters; and (3) that 10 error reports per doctor (five collected on paper forms and five on computer) was a manageable burden for participants and a reasonable number to expect in a 20 week data collection period (from 9 May to 26 September 2000). Physicians reported the first five errors they observed in their practices after the starting date. On completion of their first five reports, they made a further five reports using the alternative method of data transfer.

The data consisted of observations of family physicians during their daily clinical practice, whether in ambulatory care clinics, hospitals, patients' homes, nursing homes, or other sites of care delivery.

\section{Definition of "error"}

The definition of medical error used in this study was that adopted by the US Institute of Medicine - that is, "safety is defined as freedom from accidental injury" and "error is defined as the failure of a planned action to be completed as intended or the use of a wrong plan to achieve an aim". We made this definition functional for participating family physicians by explaining:

"For this study, please report anything that happened in your own practice that should not have happened, that was not anticipated and that makes you say 'that should not happen in my practice, and I don't want it to happen again'. It can be small or large, administrative or clinical-anything that you identify as something to be avoided in the future."

To be eligible for inclusion in the study, reported events did not require an adverse outcome of care or actual or potential harm to patients.

Collected data included information about the error and, if appropriate, affected patients. A free text description of the error was made with prompts to record what happened and observed consequences. The date, time, and site at which the error occurred and the date, time, and site at which it was recognized by the physician were also collected. If the error related to a particular patient, the report included the patient's age, sex, ethnicity, consequence to the patient and to others, and whether the reporting doctor usually provided the patient's care.

\section{Analysis of data}

The analytical plan rested on three key decisions:

(1) To "bracket" prior knowledge of other characterizations of medical errors and to conduct a qualitative analysis that allowed the taxonomy to emerge from the study data. The rationale for this decision was that the study base of family physicians' self-reports was different from the data sources used to form other taxonomies, so using them might create traps of preconception that would limit the emergence of new knowledge from the current study.

(2) To be guided in the analysis primarily by the words used in the report and to systematically challenge use of the analysts' clinical experience in interpreting these words. Sometimes this clinical experience was essential to understanding the reported event, but clinically informed "reading between the lines" was judiciously applied, debated, and documented in the process of creating the taxonomy. As far as possible, the resulting taxonomy therefore reflects the reporters' descriptions of events they recognized as "errors", rather than analysts' interpretations of "root causes" of reported events.

(3) To assign a single error classification code to each error report. Where it was clear that a number of different errors actually happened in a single reported event, we established a rule to assign the classification code that reflected only the first error in the chronology of the event. Thus we compiled a list of 330 errors from 330 reports.

One researcher (SD) initially made an immersion/ crystallization analysis ${ }^{21}$ of free text responses to develop a draft error taxonomy. Using this draft taxonomy but blinded to the error category applied to the report, two family physician researchers (DM and RP) then reviewed every report independently and assigned a category to each. Following independent review, all three reviewers debated the appropriateness of each report's assignment to specific categories and established a revised definition of categories, re-framing and reconstructing the taxonomy through consensus. Several iterations of this process resulted in the taxonomy presented in fig 1 . Fourteen reports $(4.1 \%$ of total) were not used in creating the taxonomy as they were reports of adverse events that the three reviewers agreed did not arise from any error.

As part of a taxonomy useful for research purposes but separate from the description of error types, we compiled a list of contextual factors including the difference between the date, time, and place at which the error was recognized and the date, time, and place at which the doctor reported the error occurring, and patient demographic characteristics. We also reviewed the reports for descriptions of actual harms or costs accruing to patients, doctors, staff, or the health system generally. 


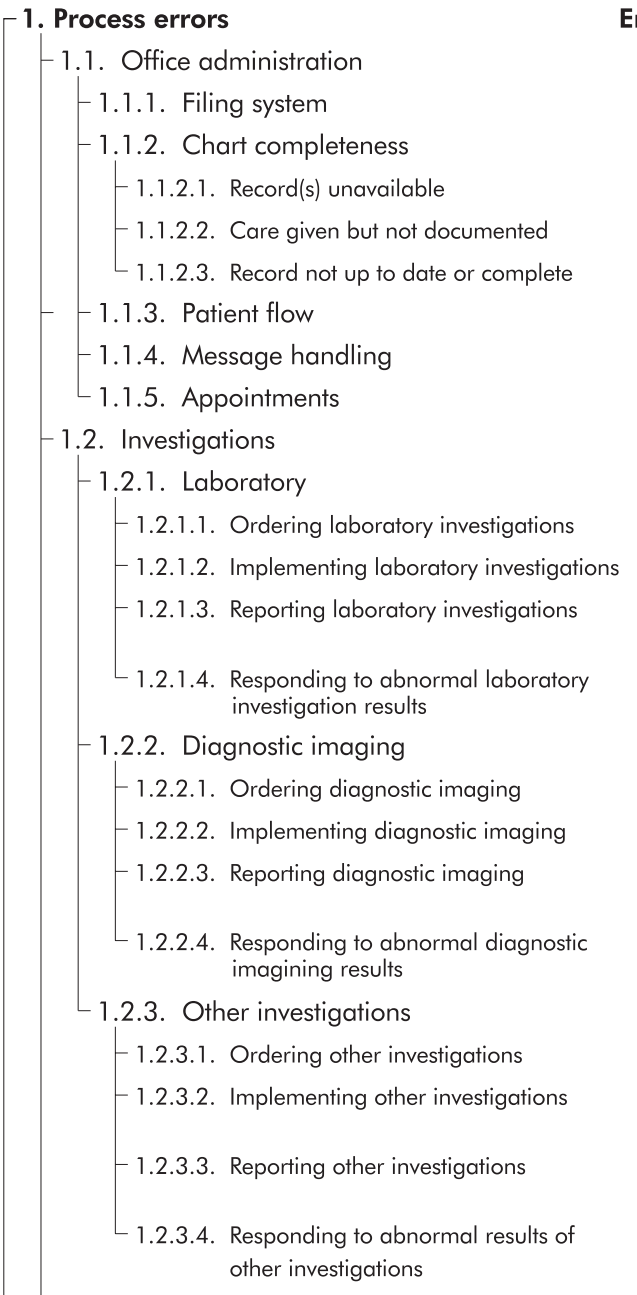

1.3. Treatments

- 1.3.1. Medications

- 1.3.1.1. Ordering medications

- 1.3.1.2. Implementing medication orders

1.3.1.3. Receiving medications

1.3.2. Other treatments

-1.3.2.1. Ordering other treatments

1.3.2.2. Implementing other treatments

1.4. Communication

- 1.4.1. Communication with patients

L 1.4.1.1. Consent errors

- 1.4.2. Communication with non-physician colleagues

1.4.3. Communication with physician colleagues

- 1.5. Payment

2. Knowledge and skills errors

2.1. Execution of a clinical task

2.2. Mis-diagnosis

2.3. Wrong treatment decision
Errors in a process of the healthcare delivery system

284

- Errors in the process of conducting an administrative task

102

- Information filed in wrong place or wrong time

- Unavailability of information that should have been in patients' charts

- Entire chart or part of chart could not be accessed when needed

- Care provided was not documented

- Item(s) of information missing from chart

- Errors in patients' movement through the healthcare delivery system

- Errors in the taking and distributing of messages

- Errors in managing appointments for healthcare

- Errors in the process of investigating a patient's condition

- Errors in the process of laboratory investigations

- Wrong test ordered or test not ordered when appropriate

- Errors in the process of obtaining or processing a laboratory specimen

- Error in the process of physician receiving accurate laboratory results in a timely fashion

- Inappropriate response to an abnormal laboratory result

- Errors in the process of diagnostic imaging investigations

- Wrong test ordered or test not ordered when appropriate

- Errors in the process of obtaining or processing of a diagnostic image

- Error in the process of physician receiving accurate results of a diagnostic image in a timely fashion

- Inappropriate response to an abnormal diagnostic image

- Errors in the process of other investigations

- Wrong test ordered or test not ordered when appropriate

- Errors in the process of obtaining or processing of other diagnostic investigation

- Error in the process of physician receiving accurate test results of other investigation in a timely fashion

- Inappropriate response to an abnormal result of other investigation

- Errors in the process of treating a patient's condition

- Errors in the process of treating with medications

- Wrong medication or wrong dose of medication ordered or medication not ordered by physician when appropriate

- Error in the process of delivering a medication order or inappropriate medication order by a provider working under physician supervision

- Error in the process of dispensing medication as ordered

- Errors in the process of treating other than by medication

- Wrong treatment ordered or treatment not ordered when appropriate

- Error in the process of providing treatment other than medication

- Errors in the process of communication

- Errors in communication between physicians and patients

- Errors in the process of obtaining informed consent

- Errors in communication between physicians and non-physician healthcare providers

- Errors in communication with physicians outside the practice

- Errors in the process of healthcare payment systems

Errors arising from lack of clinical knowledge or skills

- Errors arising during the performance of a clinical task due to a lack of clinical knowledge or skills

- Wrong or missed diagnosis

- Wrong treatment decision arising from a lack of clinical knowledge or skills 
Table 1 Demographic characteristics of affected patients $(n=328)$

\begin{tabular}{lc}
\hline Age $(n=307)$ & \\
Mean (SD) & $49.3(23.5)$ years \\
Median & 47.8 years \\
Sex & \\
Male & $138(42.1 \%)$ \\
Female & $185(56.4 \%)$ \\
Race & \\
White & $250(76.2 \%)$ \\
Black & $32(9.8 \%)$ \\
Hispanic & $20(6.1 \%)$ \\
Other & $22(6.7 \%)$ \\
Usually obtains medical care from & $234(71.3 \%)$ \\
reporting physician & \\
\hline
\end{tabular}

\section{RESULTS}

\section{Categorization of errors}

Qualitative analysis of the 330 error reports produced the four-layer taxonomy shown in fig 1 . The taxonomy comprises two primary categories which distinguish between errors attributable to aspects of care delivery systems ("Process" errors; $\mathrm{n}=284$ (86.1\% of total errors)) and errors that could only be averted by improving providers' clinical skills and/or knowledge or diverting clinical tasks to clinically trained providers ("Knowledge and skills" errors; $\mathrm{n}=46$ ( $13.9 \%$ of total)). Included in the "Knowledge and skills" category were events such as receptionists failing to make urgent appointments available for acutely ill infants as well as physicians deciding to discharge patients from hospital before they were able to function well at home. "Process" errors included administrative mistakes $(102 ; 30.9 \%)$, failure in investigation processes $(82 ; 24.8 \%)$, treatment delivery problems $(76 ; 23.0 \%)$, miscommunication $(19 ; 5.8 \%)$, and payment system mix ups (4; $1.2 \%)$. One "Process" error could not be assigned to any subcategory as the reporting physician provided insufficient details. "Knowledge and skills" errors included errors in the execution of a clinical task (19; 5.8\%), misdiagnosis (13; $3.9 \%)$, and wrong treatment decisions $(14 ; 4.2 \%)$. The eight layer II categories were further subdivided into layers III (13 categories) and IV (21 categories), providing further detail.

\section{Context}

Analysis of contextual factors showed that physicians recognized reported errors on the day of the event in only 38 cases $(11.6 \%)$, but $49.8 \%$ of errors were recognized within 2 weeks of their occurrence. The greatest reported time lapse between error occurrence and recognition was 141 days. Physicians' offices were the main sites of error occurrence $(72.3 \%)$ and recognition $(72.1 \%)$, but many other sites were implicated: 22 reports involved an error event recognized in hospitals and there were 13 reports of errors recognized in emergency departments, 10 reports of errors recognized in nursing homes, and 15 reports of errors recognized in patients' homes, pharmacies, laboratories, and the spaces between these sites (including physicians' cars). Only two error reports (failure of a refrigerator to maintain a temperature that could preserve its contents and inadequately cleaned equipment) were not linked to one unique affected patient.

The demographic characteristics of affected patients are shown in table 1 . In $74.5 \%$ of reports the event involved a patient whom the reporting physician already knew.

\section{Consequences}

Physicians reported a consequence to patients (96 reports; $29.2 \%$ ) or to someone else, including patients' families, practice staff, and themselves (66 reports; $20.2 \%$ ) in response to separate prompts. Free text responses were reviewed to
Table 2 Consequences of reported error events $(n=330)$

\begin{tabular}{lc}
\hline None & $184(55.8 \%)$ \\
Care consequences & $70(21.2 \%)$ \\
$\quad$ Care delayed & $3(0.9 \%)$ \\
Care extended & \\
Financial and time cost consequences & $29(8.8 \%)$ \\
To patients & $16(4.8 \%)$ \\
To physicians & $15(4.5 \%)$ \\
To the health system & \\
Patient health consequences & $40(12.1 \%)$ \\
Patient upset or lost trust in physician & $23(7.0 \%)$ \\
Patient became ill & $8(2.4 \%)$ \\
Patient did not regain health & $10(3.0 \%)$ \\
Patient admitted to hospital & $1(0.3 \%)$ \\
Patient died &
\end{tabular}

construct the consequence categories shown in table 2. Each case may be associated with more than one consequence, but in 184 reports $(55.8 \%)$ no consequence was recorded or implied. Physicians reported emotional distress or loss of trust by the patient in 25 reports $(7.6 \%)$. Ten errors precipitated hospital admission; one death resulted.

\section{DISCUSSION}

This analysis organizes error reports made by family physicians into a meaningful but preliminary taxonomy. The importance of the taxonomy is that it provides a tool for understanding opportunities to improve patient care and suggests priority areas for remedial attention. The foundation of this tool in primary care is important because of the current dearth of patient safety research in this setting. By virtue of volume alone, ${ }^{1622}$ primary care delivery systems probably contain substantial opportunities for better protection of patients from harm. This study shows that practising family physicians report a spectrum of medical errors that is different from the types of medical errors previously identified in hospital based care. ${ }^{4}$ Most errors reported arose from problems with healthcare processes. Medication errors were reported frequently but they were not such a dominant area of concern as one might infer from the concentration of the medical error literature on this aspect of care. ${ }^{510}{ }^{11}$ Both investigations and administrative dysfunction contributed more often to reported errors; errors in clinical knowledge or skills were also reported.

The taxonomy is preliminary but, with further use, its categories will become better defined. The anthropological research approach we took to this analysis is best satisfied when cell "saturation" is achieved. ${ }^{23}$ The small number of reports assigned to each category shown in fig 1 suggests that saturation has not been achieved in this study. Given the breadth of scope of the practice of family physicians, it may take some time and repeated use of this tool to develop it to the stage where it truly describes all or most medical errors in primary care.

The definition of error we used in this study deliberately related to true errors or mistakes, without regard to actual or potential consequences. It therefore differs from much of the existing literature on patient safety which tends to focus on "preventable adverse events" (actual harm) ${ }^{713}$ or "sentinel events" (potential harm). ${ }^{518}$ Our definition risks attracting reports of trivial events, thereby distracting from the central purpose of patient safety research-namely, to find ways to protect patients from harm. Many reports $(55.8 \%)$ may seem trivial in that no patient was harmed; however, an event that is trivial on one occasion may be non-trivial on another. The data highlight the important role of administrative systems, particularly medical record systems, as a source of errors that matter to patients and doctors. A single death was the most 
serious consequence reported in this study and was traced to a failure of message handling. The risk of death was a possibility in other cases-for instance, a physician was unable to contact a patient whose skin biopsy result indicated melanoma because there was no contact information for the patient. Both these examples defeat the notion that message handling and registration processes are unimportant in taking care of patients and securing their safety. Although most undelivered messages or missing data in patient records do not result in harm, these are such common and redeemable system failings that they deserve attention. This attention will save lives.

The definition of "error" used in this study was chosen because of its comprehensibility by participants and on the grounds that so little previous patient safety research has focused on primary care settings that there was a need to determine the type of errors seen by family physicians. Family physicians, as healthcare coordinators for patients, are well positioned to observe health system problems in a wide variety of settings. In this study many errors were observed in sites other than the physician's office and many involved other healthcare professions. By including the full scope of family practice, the study identified errors in both inpatient and outpatient settings and in the transition between care settings. The breadth of the scope of family practice was reflected in the wide variety of error types reported, the spectrum of patient characteristics represented in the data set, and the range of consequences observed. Other healthcare providers, practice staff, and patients are likely to perceive medical errors differently and the research agenda needs to be extended to include their views.

The analytical approach used in this study required key decisions that might be differently addressed by others. There were tensions in our decisions. For instance, our first decision was to eschew other taxonomies and to develop a characterization of primary care errors directly from the data, and our second was to be guided by the words in the report as we analysed each one. On some occasions the reporter showed awareness of existing taxonomies by stating "this is a medication error" or "this is a laboratory error". When this happened we remained true to our decision to follow the report's words and accepted the influence of other similar work on reporters' language. Our third decision-to assign each report to only one category-was taken to simplify the analysis. We recognize the attraction of coding all perceived errors described in reports but, if this approach is taken, concurrent consideration of chronology may be needed to make sense of the event.

We did not meet the stated goal of 50 participants. Although 54 volunteered for the study, $12(22.2 \%)$ did not send in any reports. We attribute this failure to difficulty with managing unfamiliar computer systems, but equally it might be because those doctors did not observe any errors or they feared to contribute because of concern about litigation. If we had collected the intended 500 reports rather than the actual 344 (330 errors and 14 non-error adverse events), the taxonomy might contain more categories, especially at finer categorization layers. In this early stage of understanding the nature of primary care medical errors, this preliminary taxonomy must be regarded as a work in progress.

\section{CONCLUSION}

There are important differences between care in hospitals (where most effort has been focused to improve patient safety) and primary care where most people receive care for most of their health problems. It is therefore necessary to develop a relevant taxonomy of errors as a framework for understanding threats to patient safety in primary care and as a basis for proposing remedies. This study provides a preliminary tool that may be used and developed by further primary

\section{Key messages}

- Family physicians reported medical errors in their primary care offices and across a wide variety of healthcare settings when asked to describe "things that go wrong in practice" rather than reporting observed, suspected, or potential harms to patients.

- A taxonomy of medical errors in family practice was developed which provides a tool for understanding opportunities for improving patient care and suggests priority areas for remedial attention.

- Medical errors in family practice are different from those reported in hospitals.

- Medical errors in family practice occur in administrative systems, laboratory and diagnostic imaging processes, and as a result of shorffalls in the knowledge and skills of different care providers, as well as medication errors.

- Patients affected by medical errors reported by family physicians are from all demographic groups, have both chronic and acute healthcare needs of all levels of severity, and are often well known by their family physician.

- Common and apparently trivial health system problems in primary care can sometimes harm and even kill patients.

care research. It also confirms that errors reported by practising family physicians are not the same as those recorded from hospital based datasets and that they are important to patients.

\section{ACKNOWLEDGEMENTS}

The authors acknowledge the following family physicians who contributed data for this study: Drs D Ballan, M Bonnell, L E Davis, J E Farmer, T M Fradsen, T L Hankey, M Hartsell, M Hayes, J M Herr, G S Hoekzema, K Krohn, D Madlon-Kay, G Maxted, A J Orzano, R Pallay, W Reynolds, A Selinger, P Sherrod, M Skaufle, D Smith, J Smucny, R Warhank, R O Winter, L Wollstadt, and the Utah Valley Family Practice Residency. The World Health Network developed the software and provided computers and software support for the study. We thank V Patel, B Patel, L Niebauer, L Hughes and M Lewis of the World Health Network for their efforts to ensure that physicians had software and computers to work with and for establishing the processes for secure electronic data transfer. Dr D C Iverson provided advice on study design. The American Academy of Family Physicians supported the study through its funding of the Robert Graham Center: Policy Studies in Family Practice and Primary Care and through full initial funding of the National Network for Family Practice and Primary Care Research. We are grateful to C Phillips of the American Academy of Family Physicians for managing the receipt and transfer of electronic data in Kansas and to L Smith of the Robert Graham Center and T Stewart of the National Network for their administrative support of the study.

\section{Authors' affiliations}

S M Dovey, D S Meyers, R L Phillips Jr, L A Green, G E Fryer, The Robert Graham Center: Policy Studies in Family Practice and Primary Care, Washington, DC 20036, USA

J M Galliher, J Kappus, The National Network for Family Practice and Primary Care Research, The American Academy of Family Physicians, Leawood, KS 66211-2672, USA

P Grob, Department of Postgraduate General Practice, The Postgraduate Deanery for Kent, East Surrey and Sussex, Guildford, Surrey GU2 5RF, UK

\section{REFERENCES}

1 Heath I, Evans $P$, van Weel $C$. The specialist of the discipline of general practice. BN 2000;320:326-7.

2 Donaldson M, Yordy K, Lohr K, et al. Primary care: America's health in a new era. Washington, DC: Institute of Medicine, 1996.

3 Olesen F, Dickinson J, Hiortdahl P. General practice: time for a new definition. BMV 2000;320:354-7.

4 Brennan TA, Leape LL, Laird NM, et al. Incidence of adverse events and negligence in hospitalized patients: results of the Harvard Medical Practice Study I. N Engl J Med 1991;324:370-6.

5 Bates DW, Cullen DJ, Laird N, et al. Incidence of adverse drug events and potential adverse drug events: implications for prevention. JAMA 1995;274:29-34 
6 Kohn LT, Corrigan JM, Donaldson MS. To err is human: building a safer health system. Washington, DC: National Academy Press, 1999.

7 Thomas E, Studdert D, Burstin H, et al. Incidence and types of adverse events and negligent care in Utah and Colorado. Med Care 2000;38:261-71.

8 Thomas E, Brennan T. Incidence and types of preventable adverse events in elderly patients: population based review of medical records. BM 2000;320:741-4.

9 Wilson R, Runciman W, Gibbert R, et al. The quality in Australian health care study. Med J Aust 1995:163:458-71.

10 Bates D, Teich J, Lee J, et al. The impact of computerized physician order entry on medication error prevention. J Am Med Inform Assoc 1999;6:313-21

11 Phillips D, Christenfiels N, Glynn L. Increase in US medication error deaths between 1983 and 1993. Lancet 1998:351:643-4.

12 Gaba DM. Human error in anesthetic mishaps. Int Anesthesiol Clin 1989;27: 137-47.

13 Bates D, Leape L, Petrycki S. Incidence and preventability of adverse drug events in hospitalized patients. J Gen Intern Med 1993:8:289-94.

14 McGuire H, Horsley J, Salter D, et al. Measuring and managing quality of surgery: statistical vs incidental approaches. Arch Surg 1992;127:733-7.
15 White KL, Williams TF, Greenberg BG. The ecology of medical care. N Engl J Med 1961;265:885-92

16 Green L, Fryer G, Yawn B, et al. The ecology of medical care revisited. N Engl J Med 2001:344:2021-5.

17 Britt H, Miller GC, Steven ID, et al. Collecting data on potentially harmful events: a method for monitoring incidents in general practice. Fam Pract 1997;14:101-6.

18 Bhasale A, Miller GC, Reid S, et al. Analysing potential harm in Australian general practice: an incident-monitoring study. Med J Aust 1998; 169:73-6.

19 Fischer G, Fetters M, Munro A, et al. Adverse events in primary care identified from a risk-management database. J Fam Pract 1997;45:38-9.

20 Green L. The weekly return as a practical instrument for data collection in office based research. Fam Med 1988;20:182-4.

21 Miller W, Crabtree B. Primary care research: a multimethod typology and qualitative road map. In: Doing qualitative research. Newbury Park, CA: Sage Publications Inc, 1992: 3-30.

22 Wilson T, Pringle $M$, Sheikh A. Promoting patient safety in primary care research, action, and leadership are required. BM 2001;323:583-4.

23 Patton M. Qualitative evaluation and research methods. 2nd ed. Newbury Park, CA: Sage Publications Inc, 1990.

\section{Beyond Bristol: Improving Heath Care - A Conference}

Tragedies that expose the inadequacies of health care systems make the news and quite often a public inquiry follows but then they get forgotten by all but those affected. None, however, has gripped the British health care professions and the public quite as much as the unfolding of the events linking the performance of two cardiac surgeons and the paediatric cardiac surgical services at Bristol Royal Infirmary with the outcome for children entrusted to their care. The effect of this "local" difficulty on British health care in general and the medical profession in particular has been seismic. Perhaps the anger expressed over the avoidable deaths and disability of children looked after in Bristol has jolted us all to grasp the reality that this is not a "local" issue and that the underlying problems pervade the whole health service. The public inquiry, set up after it emerged that two surgeons had a much higher operative mortality than other paediatric cardiac surgeons and that problems with that unit had been "known about" for years, has been wide ranging. Although the terms of reference were "To look into the management of children receiving complex cardiac surgery services at the Bristol Royal Infirmary between 1984 and 1995", the inquiry team was also asked to conclude by making "recommendations that could help secure high quality care across the NHS". In the end there were 198 recommendations usefully categorised under seven headings to reflect the patient journey:

- Respect and honesty

- A health service which is well led

- Competent health care professionals

- The safety of care

- Care of an appropriate standard

- Public involvement through empowerment

- The care of children

These recommendations touch every aspect of the health care system and have relevance internationally. The events at Bristol may have started as a "local" issue but the problems and the solutions are to be found within the wider system of care. We all hold some responsibility for understanding what is needed and implementing the necessary changes. Action must replace anger and anguish as the motif of this tragedy. This is one inquiry whose end must not be a dusty footnote in the tired history of failures of health care. But taking it forward will not be easy. As noted in the introduction to the inquiry: "nothing can be done in the cheap"; "there are no quick fixes" and "change can only be brought about with the willing and active participation of those involved in health care". And as the inquiry also indicates, the most significant change called for is one that does not attract a heading of its own: " a change in the culture of the NHS".

A one day conference to explore some of the many tough but crucial areas for change articulated by the inquiry has been organised jointly by the BMA, the BM Publishing Group, the Journal of Medical Ethics and Quality \& Safety in Health Care. The conference will take place on 18 November 2002 and aims to look forward and assess in the light of the report what needs to be done to: (1) improve the quality and safety of health care; (2) put the patient at the centre of health care; and (3) reduce errors. The conference will consider the lessons learnt from the Bristol Inquiry; discuss the practicality of implementing the recommended changes; and produce realistic action points that we hope will be the beginning of the long and difficult process of changing the culture of the NHS.

Venue: Queen Elizabeth II Conference Centre, Broad Sanctuary, Westminster, London SW 1P 3EE.

Closing date: Monday 11 November 2002.

Further details and a brochure are available from: BMA/BM Conference Unit, PO Box 295, London WC1H 9TE. Telephone: + 44 (0) 207383 6605; fax: + 44 (0) 207383 6663; email: confunit@bma.org.uk; website: www.bma.org.uk 